Reprod. Nutr. Dévelop., 1981, 21 (2), 237-245.

\title{
Expression du gène de thyroglobuline dans les cellules thyroïdiennes en culture : régulation par la thyrotropine
}

\author{
par Odile CHABAUD, Judith CHEBATH, J. MAUCHAMP \\ Faculté de Médecine, \\ Laborofoire de Biochimie médicale ef U 38 INSERM, \\ 27, Bd Jean Moulin, 13385 Marseille Cedex 4, France.
}

Summary. Thyroglobulin gene expression in cultured thyroid cells : regulation by thyrofropin.

The influence of thyrotropin (TSH) on thyroglobulin ( $\mathrm{Tgb}$ ) biosynthesis has been studied in porcine thyroid cells maintained in primo culture. After 4 days of culture and 1-hr labelling with ${ }^{35} \mathrm{~S}$ Met, Tgb specific radioactivity was 10 times higher in TSH-treated cells than in control cells. The levels at which TSH action occurred have been described. i) In TSH cell extracts, 70 p. 100 of the Tgb mRNA sequences, quantified by hybridization to a specific ${ }^{3} \mathrm{H}$ cDNA probe, were located in highly polymeric polysomes, while 70 p. 100 of the Tgb mRNA in the control extracts were in light polysomes. This suggests that TSH specifically or nonspecifically stimulates the initiation of translation on $\mathrm{Tgb}$ mRNA. ii) The relative amount of Tgb mRNA in the cytoplasm of TSH-treated cells was 3 times higher than in the control cells. This correlates with the fact that $\mathrm{Tgb}$ biosynthesis relative to fotal protein synthesis increased similarly, indicating that TSH controls relative $\mathrm{Tgb}$ biosynthesis by specifically regulating cytoplasmic Tgb mRNA content. iii) The nuclear RNA of TSH-treated cells contained 2.2 times more Tgb mRNA sequences than the nuclear RNA of control cells, showing that TSH specifically regulates Tgb gene expression at the nuclear level (transcription or stabilization).

\section{Introduction.}

La régulation de la synthèse de protéines spécifiques par les hormones polypeptidiques est encore mal connue. Quelques exemples ont été décrits où les hormones polypeptidiques contrôlent l'accumulation d'ARN messagers spécifiques : ainsi en est-il de la prolactine pour l'ARNm de caséine (Matusik ef Rosen, 1978 ; Devinoy, Houdebine et Delouis, 1978) ou de l'hormone de croissance pour les ARNm d'albumine (Keller et Taylor, 1979) et d' $\alpha_{2, u}$ globuline (Roy et Dowbenko, 1977). La thyroglobuline (Tgb), précurseur des hormones thyroïdiennes, glycoprotéine de PM 660000 composée de 2 sous-unités de PM 330000 , est majoritaire dans la glande thyroïde. La thyrotropine (TSH) hormone polypeptidique hypophysaire contrôle la fonction thyroiddienne et son premier effet est l'augmentation du cAMP intracellulaire (Dumont, 1971). Une régulation spécifique de la synthèse de Tgb par la TSH n'a pas été clairement démontrée bien que, dans quelques expériences de privation de TSH, l'incorporation d'acides 
aminés marqués dans la Tgb diminue préférentiellement (Pavlovic-Hournac, Rappaport et Nunez, 1971 ; Regard et Mauchamp, 1971 ; Pavlovic-Hournac et Delbauffe, 1973).

Le travail présenté ici montre que la TSH est susceptible d'intervenir à plusieurs niveaux pour réguler l'expression du gène de Tgb dans un système de cellules thyroïdiennes de porc maintenues en primoculture. Préalablement l'ARNm de Tgb de thyroïde de mouton a éfé purifié et caractérisé (Chebath et al., 1977a, b) et transcrit en ADN complémentaire radioactif (Chabaud, Chebath et Jacquet, 1980). Celui-ci a servi de sonde pour évaluer les quantités d'ARNm de Tgb dans les cellules thyroïdiennes de porc cultivées en présence ou en absence de TSH (Chebath, Chabaud et Mauchamp, 1979).

\section{Matériel et méthodes.}

Culture de cellules. - Les cellules thyroïdiennes de porc sont isolées et cultivées en suspension (Mauchamp ef al., 1979) en absence (cellules témoins) ou en présence de $\mathrm{TSH}(0,05$ ef $5 \mathrm{mU} / \mathrm{ml})$.

Incorporation de ${ }^{35} \mathrm{~S}$-méthionine dans les protéines ef la Tgb dans les cellules thyroïdiennes de porc en culfure. - Après 4 jours de culture avec ou sans TSH les cellules sont incubées pendant $1 \mathrm{~h}$ en présence de ${ }^{35}$ S-méthionine (Chabaud et al., 1980).

Les radioactivités totales, préci pitables par l'acide trichloracétique ef précipitables par un sérum antithyroglobuline de porc sont déterminées sur les surnageants $27000 \times g$ des homogénats (Chabaud ef al., 1980). Les surnageants $27000 \times \mathrm{g}$ sont analysés en gradient de saccharose 15-50 p. 100 (p/v) (Chabaud ef al., 1980). La méthode de Mc Intire et Sproull, 1957, a été utilisée pour l'évaluation quantitative du DNA.

Extraction des ARNs cytoplasmiques et des ARNs nucléaires. - L'ARN cytoplasmique est extrait à partir de surnageant $27000 \times g$ d'homogénats de cellules (Chebath, Chabaud et Mauchamp, 1979). L'ARN nucléaire est obtenu à partir de noyaux purifiés (Tsai ef al., 1978) par traitements SDS-phénol-chloroforme, puis DNase I (Worthington DPFF, préalablement traitée à l'iodoacétate ; Zimmerman et Sandeen, 1966) selon la méthode de Roop ef al. (1978).

Evaluation de la concentration en ARNm de Tgb par hybridation avec ${ }^{\prime 3} \mathrm{H}$ ADNc de Tgb de mouton. - La concentration en ARNm de Tgb dans l'ARN cytoplasmique est déterminée par cinétiques d'hybridation en excès d'ARN (Chebath, Chabaud et Mauchamp, 1979). Des quantités variables d'ARN nucléaire sont incubées avec l'ADNc pendant $16 \mathrm{~h}$ à $65^{\circ} \mathrm{C}$ dans les conditions décrites (Chabaud, Chebath ef Jacquet, 1980) pour évaluer le contenu relatif en ARNm de Tgb.

\section{Résultats.}

L'effet de la TSH sur la synthèse de Tgb a été observé successivement dans le cytoplasme et dans le noyau de cellules thyroïdiennes de porc cultivées en absence ou en présence de TSH $(0,05$ et $5 \mathrm{mU} / \mathrm{ml})$ pendant 4 jours. 
Effet de la TSH au niveau cytoplasmique : traduction ef taux d'ARNm de Tgb.

Les cellules thyroïdiennes cultivées avec et sans TSH sont incubées $1 \mathrm{~h}$ avec différentes concentrations de méthionine froide, l'activité spécifique de la ${ }^{35} \mathrm{~S}$-méthionine restant constante. On sait en effet que l'incorporation d'acides aminés radioactifs peut être dépendante de la concentration en acides aminés dans le milieu extracellulaire comme Seglen (1976), par exemple, l'a montré pour les hépatocytes isolés. La TSH augmente l'incorporation de ${ }^{35} \mathrm{~S}$-méthionine dans les protéines même à faible concentration en méthionine. Cet effet est du même ordre de grandeur quelle que soit la concentration en méthionine (fig. 1a). Avec la concentration en méthionine du milieu

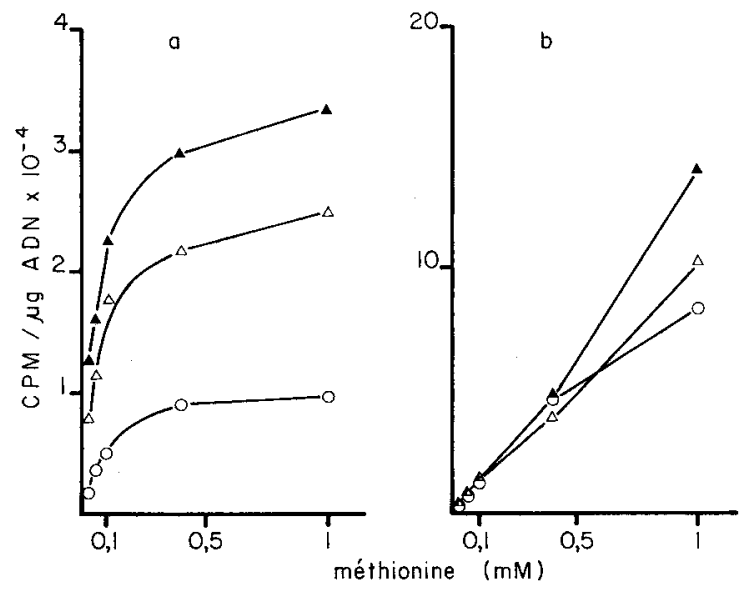

FIG. 1. - Radioactivités ${ }^{35} \mathrm{~S}$-méthionine incorporées dans les proféines ef radioactivités libres en fonction de la concentration en méthionine froide dans le milieu extra-cellulaire. La radioactivité spécifique ${ }^{33} \mathrm{~S}$ méthionine est constante $(0,4 \mathrm{mCi} / \mathrm{umole})$ et les concentrations des autres acides aminés sont celles du milieu de culture. (a) La radioactivité insoluble dans l'acide trichloracétique chaud est mesurée sur des aliquotes en double des surnageants $27000 \times \mathrm{g}$; (b) la radioactivité libre est la différence entre la radioactivité totale et fa radioactivité incorporée dans les protéines. Cellules cultivées en absence de TSH $(0)$, cellules cultivées en présence de TSH $0,05 \mathrm{mU} / \mathrm{ml}(\Delta)$ ou $5 \mathrm{mU} / \mathrm{ml}(\Delta)$. L'ADN a été mesuré suivant la méthode de Mc Intire et Sproull (1957).

TABLEAU 1

Radioactivités ${ }^{35}$-méthionine incorporées dans les protéines et les peptides de thyroglobuline dans les cellules thyroïdiennes de porc en culture

\begin{tabular}{|c|c|c|}
\hline $\begin{array}{l}\text { activité préci } \\
\text { ps. } \min ^{-1} / \mu \mathrm{g}\end{array}$ & $\begin{array}{l}\text { Radioactivité incorporée } \\
\text { dans la thyroglobuline } \\
\text { (coups. min }{ }^{-1} / \mu g \text { ADN) }\end{array}$ & $\begin{array}{l}\text { Synth } \\
\text { de th }\end{array}$ \\
\hline
\end{tabular}

\begin{tabular}{|c|c|c|c|}
\hline $\begin{array}{l}\text { Cellules témoins. . . . . } \\
\text { Cellules TSH }\end{array}$ & 9563 & 297 & 3,1 \\
\hline $\begin{array}{l}0,05 \mathrm{mU} / \mathrm{ml} \ldots \ldots \\
5 \ldots \ldots \ldots\end{array}$ & $\begin{array}{l}33456 \\
49855\end{array}$ & $\begin{array}{l}3574 \\
5305\end{array}$ & $\begin{array}{l}10,7 \\
10,6\end{array}$ \\
\hline
\end{tabular}

La radioactivité insoluble dans l'acide trichloracétique chaud et la radioactivité précipitée par un sérum antithyroglobuline de porc sont mesurées sur des aliquotes en double des surnageants $27000 \times \mathrm{g}$. Les résultats présentés sont la moyenne de 3 expériences. 
de culture $(0,1 \mathrm{mM})$, la quantité de radioactivité incorporée dans les protéines est de 3 à 4 fois plus élevée dans les cellules cultivées en présence de TSH (Chabaud et al., 1980) (tabl. 1). En absence de TSH, la diminution de la radioactivité protéique ne peut pas être due à une plus faible quantité d'acides aminés disponibles, puisque la radioactivité intracellulaire libre (différence entre la radioactivité totale et la radioactivité protéique) présente des valeurs similaires que les cellules soient cultivées avec ou sans TSH (fig. 1b). De plus, cetfe diminution ne s'explique pas seulement par la baisse d'ARN cytoplasmique (15 à 20 p. 100) dans les cellules témoins où le taux de poly(A) est le même que dans les cellules cultivées en présence de TSH (Chebath, Chabaud et Mauchamp, 1979). Il semblerait donc que l'absence de TSH entraîne une diminution de l'efficacité de la traduction.
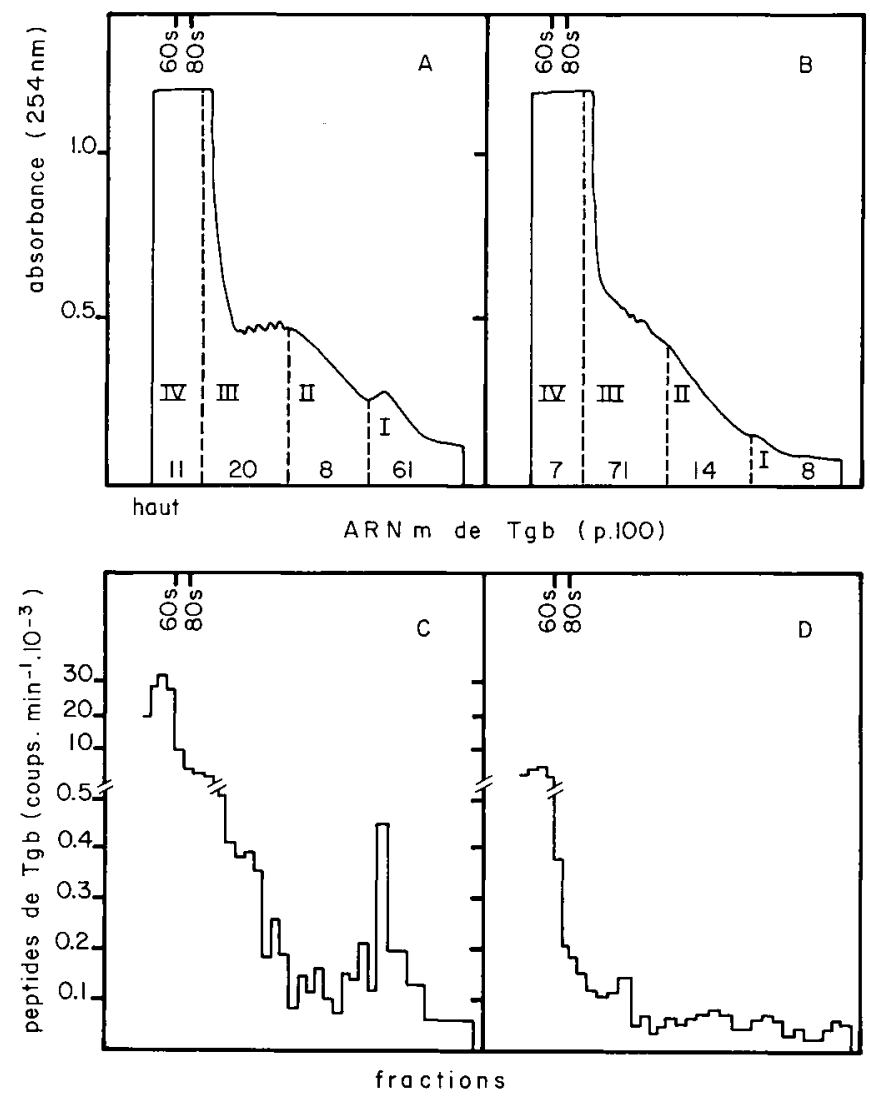

FIG. 2. - Profil des polysomes, distribution des peptides de Tgb et de l'ARN de Tgb dans les surnageants $27000 \times g$ analysés en gradient de saccharose. Les surnageants $27000 \times g$ provenant de cellules cultivées en présence de TSH $(5 \mathrm{mU} / \mathrm{ml})(A, C)$ et des cellules cultivées sans TSH (B, D) sont analysés sur gradients de saccharose. A, B : les surnageants proviennent de $24.10^{6}$ cellules. Le contenu en ARNm de Tgb est mesuré par hybridation avec l'ADN complémentaire radioactif. L'ARNm de Tgb présent dans chaque lot de fractions regroupées est exprimé en pourcentage de la quantité totale d'ARNm de Tgb du gradient. C, D : les peptides de Tgb sont mesurés dans chaque fraction par immunoprécipitation (les surnageants proviennent de $7,5.10^{6}$ cellules). 
La radioactivité incorporée dans les peptides de Tgb est 12 fois plus élevée dans les cellules cultivées en présence de TSH (tabl. 1) (Chabaud et al., 1980) alors que la synthèse totale est seulement 3 à 4 fois plus élevée. Ceci suggère qu'il existe un processus de contrôle spécifique pouvant s'exercer au niveau de la traduction ou de la quantité d'ARNm de Tgb.

En présence de TSH, les surnageants $27000 \times \mathrm{g}$ présentent un pic de polysomes lourds spécifiques de la Tgb (fig. 2A, C), pic à peine perceptible en absence de TSH (fig. 2B, D) (Chabaud ef al., 1980). En présence de TSH, 61 p. 100 des séquences d'ARNm de Tgb se trouvent associées aux polysomes lourds. En absence de TSH 7 p. 100 seulement des séquences d'ARNm de Tgb sont dans cette fraction, mais 70 p. 100 sont associées aux polysomes légers. Ce déplacement des séquences d'ARNm de Tgb vers les zones légères du gradient suggère soit une dégradation de l'ARNm de Tgb soit une diminution de la charge en monoribosomes sur les molécules d'ARNm de Tgb infactes. L'analyse des peptides de Tgb synthétisés, marqués par la ${ }^{35} \mathrm{~S}$-méthionine est en faveur de la $2^{\mathrm{e}}$ hypothèse puisque pour les 2 types de cellules, le composant majeur des immunoprécipités est la chaîne complèłe 330000 (Chabaud ef al., 1980). II semble donc que dans les cellules cultivées en absence de TSH, les molécules d'ARNm
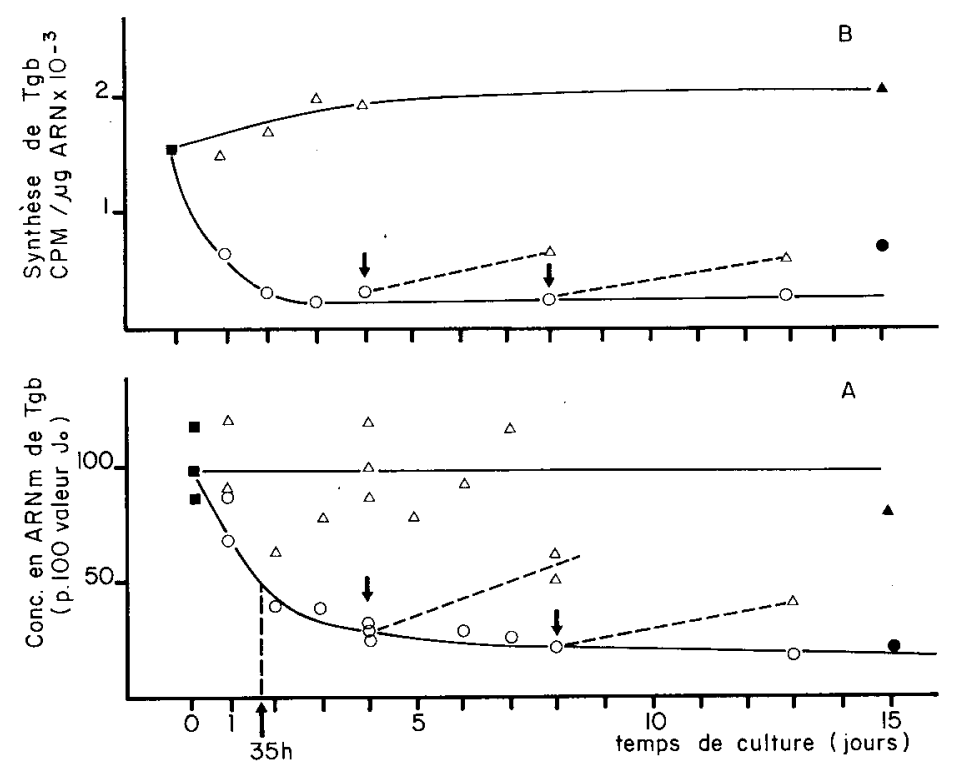

FIG. 3. - Evaluation de la quantité d'ARNm de Tgb dans le cytoplasme de cellules thyroïdiennes cultivées avec et sans TSH en fonction du temps de culfure. Le contenu en ARNm de Tgb est évalué dans les cellules fraîchement isolées ( $a$ ) et dans les cellules cultivées en absence ( 0 ) ou en présence $(\Delta)$ de TSH $(0,05$ ou $5 \mathrm{mU} / \mathrm{ml})$, par les valeurs des $R_{0} t 1 / 2$ des cinétiques d'hybridations mesurés à différents temps ( $A$, plusieurs expériences) et par l'activité messagère de l'ARN cytoplasmique mesurée en lysat de réticulocytes (B, expérience type suivant les conditions décrites, Chebath et al., $1977 b$ ). Les symboles $\Delta$ et - représentent une expérience où les cellules ont été cultivées en présence d'hydrocortisone $(1 \mu \mathrm{g} / \mathrm{ml})$ et TSH $5 \mathrm{mU} / \mathrm{ml}$. Au temps indiqué par les flèches, la TSH a été ajoutée $(5 \mathrm{mU} / \mathrm{ml})$ après changement de milieu à des cellules témoins. L'ARN a été mesuré suivant la technique décrite (Chebath, Chabaud, Mauchamp, 1979). 
de Tgb ne portent pas leur charge maximale en ribosomes, ce qui suggère une diminution de la vitesse d'initiation. L'effet de la TSH sur la vitesse d'initiation est vraisemblablement un effet général sur la synthèse protéique, mais une régulation spécifique de la traduction de l'ARNm de Tgb ne peut être exclue.

Le contrôle spécifique de l'expression du gène de Tgb par la TSH peut s'exercer sur les niveaux d'ARNm de Tgb dans le cytoplasme ou/et dans le noyau. La quantité d'ARNm de Tgb a été évaluée dans le cytoplasme des 2 types de cellules par hybridation avec la sonde ${ }^{3} \mathrm{H}$ ADN complémentaire et par traduction dans un lysat de réticulocytes (fig. 3) (Chebath, Chabaud et Mauchamp, 1979). De 0 à 15 jours de culture en présence de TSH, le contenu relatif d'ARNm de Tgb reste constant. Ce contenu diminue progressivement en absence de TSH. A 4 jours de culture, le contenu relatif d'ARNm de Tgb est 3 fois plus élevé dans le cyłoplasme des cellules cultivées avec TSH (Chebath, Chabaud et Mauchamp, 1979). Ce rapport est en bonne corrélation avec la synthèsè relative de Tgb (tabl. 1) signifiant que la TSH régule le taux de synthèse de Tgb par l'intermédiaire du niveau d'ARNm cytoplasmique.

\section{Effet de la TSH au niveau nucléaire.}

Les résultats précédents ne permettent pas d'établir si la régulation de la concentration en ARNm de Tgb a lieu dans le cytoplasme ou dans le noyau. Si après 4 ou 8 jours de culture en absence de TSH, on rajoute de la TSH pendant 4 ou 5 jours, le contenu relatif d'ARNm de Tgb dans le cytoplasme remonte (fig. 3) (Chebath, Chabaud et Mauchamp, 1979). L'augmentation du taux d'ARNm de Tgb cytoplasmique est le signe soit d'une stabilisation du messager dans le cytoplasme, soit d'un contrôle au niveau nucléaire. Déterminé par hybridation des ARNs nucléaires avec l'ADNc de Tgb, le contenu relatif en ARNm de Tgb dans le noyau est 2,2 fois plus élevé dans les cellules cultivées avec TSH pendant 4 jours que dans les cellules témoins (fig. 4). A ce

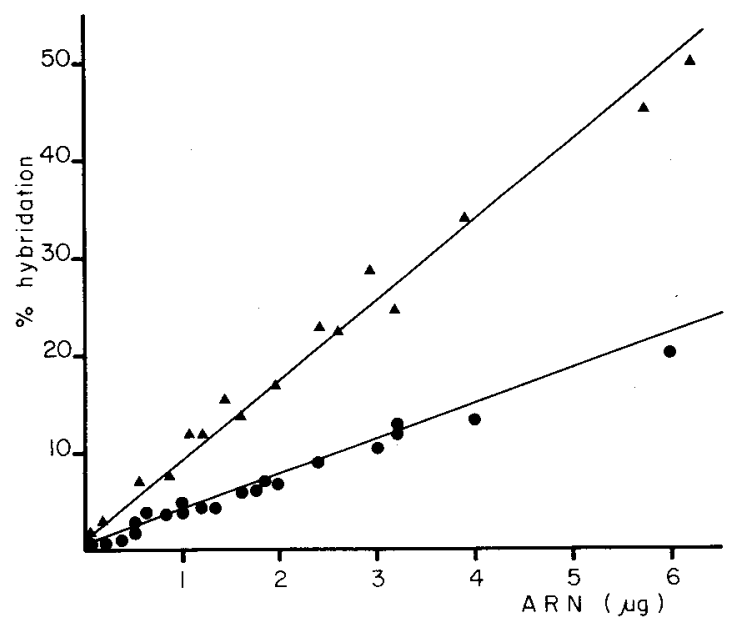

FIG. 4. - Evaluation de la quantité d'ARNm de Tgb dans les noyaux des cellules cultivées ovec ou sans TSH. Les hybridations ont été faites à temps constant avec des quantités croissantes d'ARN nucléaire provenant de cellules cultivées en absence ( $(\bullet)$ ou en présence ( $\bullet$ ) de TSH $(0,05 \mathrm{mU} / \mathrm{ml})$. Ces valeurs sont celles de 2 expériences. 
temps de culture, le rapport ARN nucléaire $(\mu \mathrm{g}) / \operatorname{ADN}(\mu \mathrm{g})$ est le même pour les deux types de cellules (environ 0,28 ). Il existe donc au niveau des noyaux une modulation spécifique du contenu en ARNm de Tgb.

\section{Discussion.}

L'influence de la TSH sur la biosynthèse de la Tgb a été étudiée dans les cellules thyroïdiennes de porc en primoculture. En absence de TSH, la synthèse protéique totale est diminuée et les résultats suggèrent que la vitesse d'initiation de la traduction de l'ARNm de Tgb est plus faible.

De plus, le niveau d'ARNm de Tgb dans le cytoplasme décroît au cours du temps de culture, tandis que ce niveau est maintenu dans le cytoplasme des cellules traitées par la TSH. L'augmentation du contenu en ARNm de Tgb cytoplasmique, que l'on peut constater après un traitement des cellules témoins par la TSH, démontre que cette hormone a un effet spécifique sur l'expression du gène de Tgb. La concentration en séquences ARNm de Tgb est plus élevée dans le noyau de cellules cultivées en présence de TSH que dans celui des cellules témoins. Ceci met en évidence que la régulation primaire a lieu au niveau des ARNs nucléaires par des modifications des vitesses de transcription et/ou de la stabilité du transcript. Une régulation au niveau cyłoplasmique n'est pourtant pas exclue. En effet le rapport des concentrations en ARNm de Tgb entre les 2 types de cellules est plus faible pour le noyau $(2,2)$ que pour le cytoplasme (3 à 4). Cette différence peut être expliquée soit par une stabilité plus grande des ARNm de Tgb dans le cytoplasme soit par le fait que les ARNs nouvellement transcrits sont exportés plus rapidement vers le cytoplasme dans les cellules cultivées en présence de TSH. Une action au niveau de plusieurs étapes du métabolisme de l'ARNm a été mise en évidence dans de nombreux systèmes de régulation hormonale de gènes spécifiques (Guyette, Matusik et Rosen, 1979 ; Hynes et al., 1979).

Les résultats présentés ici amènent à poser la question suivante : quelles sont les étapes intermédiaires entre les événements primaires (fixation de l'hormone sur la membrane plasmique) et l'action spécifique sur l'expression du gène de la thyroglobuline? L'interaction de la TSH avec son récepteur stimule les activités adénylate cyclase ef protéine-kinase CAMP dépendante, en particulier au niveau du noyau (Spaulding et Schubart, 1978). Le niveau d'AMPc régulerait de façon spécifique l'expression du gène de Tgb, par modulation du niveau de l'ARNm, comme il le fait pour un enzyme du foie (lynedjian et Hanson, 1977). D'autres hypothèses peuvent être formulées. L'information parviendrait de la membrane plasmique au noyau par des modifications des microtubules et/ou microfilaments (Revue dans Houdebine et Djiane, 1980). Enfin, il faut noter que, dans le système de culture de cellules utilisé ici, les cellules cultivées en présence de TSH sont réassociées en follicules où le pôle basal est tourné vers le milieu extérieur, tandis qu'en absence de TSH, c'est le pôle apical des cellules qui est en contact avec le milieu (Mauchamp et al., 1979). Cette différence d'organisation pourrait être partiellement impliquée dans les effets de la TSH que nous avons observés. 


\section{References}

CHABAUD O., CHEBATH J., GIRAUD A., MAUCHAMP J., 1980. Modulation by thyrotropin of thyroglobulin synthesis in cultured thyroid cells : Correlations with polysome profile and cytoplasmic thyroglobulin mRNA confent. Biochem. biophys. Res. Commun., 93, 118-126.

CHABAUD O., CHEBATH J., JACQUET M., 1980. Subcytoplasmic distribution of thyroglobulin mRNA in normal sheep thyroid. Eur. J. Biochem., 104, 125-136.

CHEBATH J., CHABAUD O., BECAREVIC A., CARTOUZOU G., LISSITZKY S., 1977a. Thyroglobulin messenger ribonucleic acid translation in vitro. Eur. J. Biochem., 77, 243-252.

CHEBATH J., CHABAUD O., BERGE-LEFRANC J. L., CARTOUZOU G., LISSITZKY S., $1977 b$. Molecular weight of the thyroglobulin messenger RNA of sheep thyroid gland. Biochem. biophys. Res. Commun., 79, 267-273.

CHEBATH J., CHABAUD O., MAUCHAMP J., 1979. Modulation of thyroglobulin messenger RNA level by thyrotropin in cultured thyroid cells. Nucl. Ac. Res., 6, 3353-3367.

DEVINOY E., HOUDEBINE L. M., DELOUIS C., 1978. Role of prolactin and glucocorticoids in the expression of casein genes in rabbit mammary gland organ culture. Quantification of casein mRNA. Biochim. biophys. Acta, 517, 360-366.

DUMONT J. E., 1971. The action of thyrotropin on thyroid metabolism, 287-412. In HARRIS R. S., MUNSON P. L., DICFALUSY E., GLOVER J., Vitamins and hormones, vol. 29, Acad. Press, New York.

GUYETTE W. A., MATUSIK R. J., ROSEN J. M., 1979. Prolactin-mediated transcriptional and posttranscriptional control of casein gene expression. Cell, 17, 1013-1023.

HOUDEBINE L. M., DJIANE J., 1980. Effects of lysomotropic agents and of microfilament and microtubule - disrupting drugs on the activation of casein-gene expression by prolactin in the mammary gland. Mol. cell. Endocrinol., 17, 1-15.

HYNES N. E., GRONER B., SIPPEL A. E., JEEP S., WURTZ T., NGUYEN-HUU M. C., GIESECK K., SCHUTZ G., 1979. Control of cellular content of chicken egg white protein specific RNA during estrogen administration and withdrawal. Biochemistry, 18, 616-624.

IYNED JIAN P. B., HANSON R. W., 1977. Increase in level of functional messenger RNA coding for phospho-enol pyruvate carboxy-kinase (GTP) during induction by cyclic adenosine $3^{\prime}$ : 5'monophosphate. J. biol. Chem., 252, 655-662.

KELLER G. H., TAYLOK J. M., 1979. Effect of hypophysectomy and growth hormone treatment on albumin mRNA levels in the rat liver. J. biol. Chem., 254, 276-278.

MCINTIRE F. C., SPROULL M. F., 1957. A simple method for determination of desoxypentose nucleic acid in tissue culfures. Proc. Soc. exp. Biol. Med., 95, 458-462.

MATUSIK R. J., ROSEN J. M., 1978. Prolactin induction of casein mRNA in organ culture. J. biol. Chem., 253, 2343-2347.

MAUCHAMP J., MARGOTAT A., CHAMBARD M., CHARRIER B., REMY L., MICHEL-BECHET M., 1979. Polarity of three-dimensional structures derived from isolated hog thyroid cells in primary culture. Cell Tiss. Res. 204, 417-430.

PAVLOVIC-HOURNAC M., DELBAUFFE D., 1973. Action of TSH on the in vivo incorporation of labeled amino acid into thyroglobulin and other thyroidal proteins. Endocrinology, 92, 12731276.

PAVLOVIC-HOURNAC M., RAPPAPORT L., NUNEZ J., 1971. Incorporation of labeled aminoacid into protein by thyroid glands from hypophysectomized rats I. In vifro studies. Endocrinology, 89, 1477-1484.

REGARD E., MAUCHAMP J., 1971. Ultrastructure de la glande thyroïde du xénope larvaire normal ef hypophysectomisé : corrélations avec la biosynthèse de la thyroglobuline. J. ultrastruct. Res., 37, 664-678.

ROOP D. R., NORDSTROM J. L., TSAI S. Y., TSAI M. J., O'MALLEY B. W., 1978. Transcription of structural and intervening sequences in the ovalbumin gene and identification of potential ovalbumin mRNA precursors. Cell, 15, 671-685.

ROY A. K., DOWBENKO D. J., 1977. Role of growth hormone in the multihormonal regulation of mRNA for $\alpha_{2^{u}}$ globulin in the liver of hypophysectomized rats. Biochemistry, 16, 3918-3922. 
SEGLEN P. O., 1976. Incorporation of radioactive aminoacids into protein in isolated rat hepatocytes. Biochim. biophys. Acta, 442, 391-404.

SPAULDING S. W., SCHUBART U. K., 1978. Time course of thyrotropin-dependent protein phosphorylation in thryroid slices. Endocrinology, 103, 2334-2341.

TSAI S. Y., ROOP D. R., TSAI M. J., STEIN J. P., MEANS A. R., O'MALLEY B. W., 1978. Effect of estrogen on the gene expression in the chick oviduct. Regulation of the ovomucoid gene. Biochemistry, 17, 5773-5780.

ZIMMERMAN S. B., SANDEEN G., 1966. The ribonuclease activity of crystallized pancreatic deoxyribonuclease. Anal. Biochem. 14, 269-277. 